\title{
Development of a Compact, Highly-sensitive and Low-cost Biological Monitoring Method using Protozoa for Detecting Toxicants in Aquatic Environment
}

\author{
Chisato Yoshimura ${ }^{1}$, Mayumi Kobayashi ${ }^{2}$, SM Mostafa Kamal Khan ${ }^{3}$, \\ MD Shafiqul Islam ${ }^{4}$, Sayaka Matsubara ${ }^{5}$, Lin Chen ${ }^{6}$, Rina Higuchi $^{7}$, Toshinobu Suzaki ${ }^{8}$ \\ ${ }^{1}$ Center for Environmental Management, Kobe University, Nada-ku, Kobe, Japan, \\ ${ }^{2,7,8}$ Department of Biology, Graduate School of Science, Kobe University, Nada-ku, Kobe, Japan, \\ ${ }^{3}$ Department of Biochemistry and Microbiology, North South University, Dhaka, Bangladesh, \\ ${ }^{4}$ Ans-VDP, Bangladesh, ${ }^{5}$ Kansai Kako Co. Ltd., Osaka, Japan, \\ ${ }^{6}$ Division of Developmental Biology, Department of Anatomy, Kyoto Prefectural University of Medicine, Kyoto, Japan.
}

\begin{abstract}
A novel method for biological monitoring to detect toxic substances in water was developed by using the protozoan Raphidiophrys contractilis as an indicator organism. In this system (named HELIOSENSOR), the adhesion of $R$. contractilis to the substratum was used as a measure of the health of the living organisms. A flow-through type chamber was designed for toxicity testing, in which cells that had been damaged by harmful materials were flushed away by the water flow. The number of protozoa was continuously monitored with a digital camera. The test results revealed that this monitoring system has high durability and efficiency compared with other bio-monitoring systems, enabling us to make a quicker and easier detection of toxic substances. This system showed particularly high sensitivity to heavy metals such as mercury,

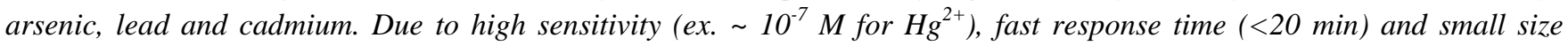
$(30 \times 14 \times 20 \mathrm{~cm})$, this system has distinct advantages over other conventional biomonitoring systems using multicellular animals such as fish and crustaceans.
\end{abstract}

Keywords—biomonitoring, heliozoa, protist, water quality control.

\section{INTRODUCTION}

Many attempts have been reported so far for monitoring water quality by using various kinds of living organisms. For example, aquatic insects and fish have been used in practice as monitor organisms to detect changes in water quality by detecting alteration in their swimming behavior. In these systems, problems are still remained to be solved as follows: 1) maintenance of the monitor organisms, 2) constructing hardware to detect movement of test organisms in high accuracy, and 3) low sensitivity and possible adaptation of the animals to the pollutants. To overcome these problems, development of a novel system of bio-monitoring was attempted by using protozoa as monitor organisms. Benthic protozoans are suitable for this purpose, because 1) most of the protozoan species can be cultured easily and inexpensively in a short time, 2) The space required to monitor their behavior is small, and 3) adaptation of the organisms to the aquatic environment does not usually occur, since protozoans are unicellular organisms. Basic investigations have demonstrated that heliozoans are most adequate protozoa, since they are highly sensitive to aquatic pollutants such as heavy metals [1], and their movement is restricted only in a planar layer [2] which allows us to use easier algorithm for automatic image analysis.
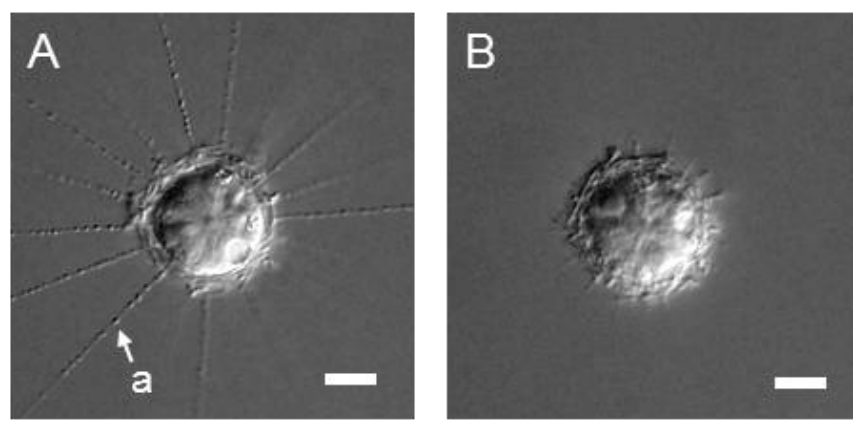

FIGURE 1. Light micrographs of Raphidiophrys contractilis showing axopodial retraction induced by treatment with 1 $\mathrm{mM} \mathrm{HgCl}_{2}$ for $5 \mathrm{~min}$. $R$. contractilis is a protozoan living in brackish water with a cell diameter of $20-30 \mu \mathrm{m}$. It is usually adhered to the bottom of water by attaching tips of axopodia (a) extending radially from the spherical cell body. A: An individual whose axopodia is fully extended. B: After $\mathbf{H g}^{2+}$ treatment with retracted axopodia. Scale bars indicate $10 \mu \mathrm{m}$. 

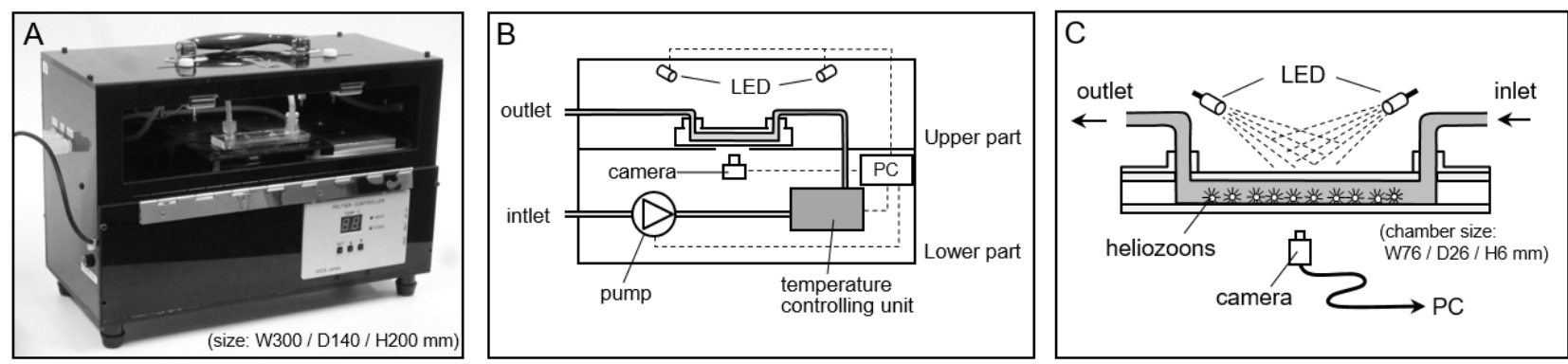

FIGURE 2. A micrograph and schematic representations of the monitoring system developed in this study. The monitoring system consists of two parts. The upper part has a monitoring chamber and a pair of LED lights. The lower part has a video camera, a pump, and a temperature controlling unit. The number of cells in the chamber is continuously monitored by image processing software. A: a photograph of the device. B: a schematic drawing of the device.

C: a detailed diagram of a flow-through type measuring chamber installed in the upper part of the device.

Heliozoans possess long and thin tentacles called axopodia radiating from the spherical cell body, in which bundles of microtubules are present as a supporting cytoskeleton. The heliozoans paralyze and capture prey organism with the aid of the axopodia [3]. The axopodia show frequent shortening and re-elongation, as their microtubules are highly sensitive to environmental factors including toxic chemical substances. We have previously examined the effect of various concentrations of heavy metal ions (zinc, lead, copper, mercury and cadmium) to the axopodia and found that the length of axopodia decreased in a concentration-dependent manner [1,4]. Raphidiophrys contractilis is one of the members of heliozoa that inhabits fresh or brackish water. This species can be cultured with ease in a bacteria-free condition [5]. In response to various environmental factors, $R$. contractilis shows axopodial retraction in a reversible fashion. The axopodia re-elongate to the original length of about $100 \mu \mathrm{m}$ in about $20 \mathrm{~min}$, when the environmental condition is fully restored. The factors include harmful chemicals such as heavy metals, changes in $\mathrm{pH}$ and water temperature, etc. In this research, we attempted to utilize such features of $R$. contractilis for water quality monitoring.

\section{MATERIAL AND METHOD}

Raphidiophrys contractilis (Fig. 1) was initially collected from a pond in Shukkeien Gardens in Hiroshima City, Japan, and cultured monoxenically in $10 \%$ artificial sea water (ASW) with the unicellular algae Chlorogonium capillatum as a sole food source [6]. Cells were harvested one week after inoculation, and introduced into a flow-through type measuring chamber as shown in Fig. 2C. The chamber was left to stand still overnight to allow the cells to settle firmly to the bottom of the chamber.

The biomonitoring system prototyped in this research (HELIOSENSOR) consisted of a device and a controlling PC. As shown in Fig. 2A, the size of the whole device was $30 \times 14 \times 20 \mathrm{~cm}$ (width, depth, and height), and has a weight of about 5 $\mathrm{kg}$. It is far smaller and lighter than other similar devices [8-10], and it can be easily carried anywhere.

The interior was divided into an upper and a lower part as shown in Figs. 2A and B. The monitoring chamber (Fig. 2C) was placed in the upper part and on top of the camera, and still images of the heliozoans attached to the bottom surface of the chamber were taken at constant time intervals. In the lower part of the device, a small pump for introducing the test water and a temperature controlling device (Peltier type) for keeping the water temperature at $20 \pm 1{ }^{\circ} \mathrm{C}$ were installed. The chamber was illuminated obliquely using a highly directional LED light source, by which a dark-field effect was obtained for yielding highly-contrasted pictures of the heliozoans as shown in Fig. 3. The photographed images were sent to a PC, and the number of cells located in a measuring area $(2 \times 2 \mathrm{~mm})$ was continuously monitored.
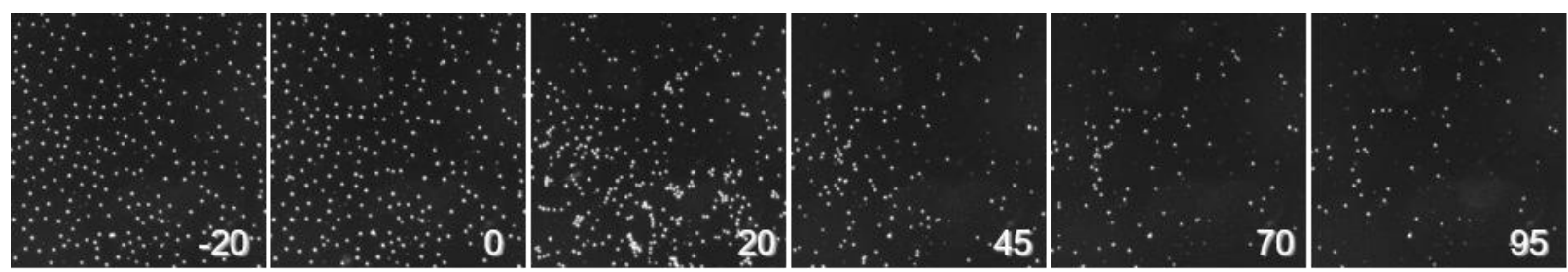

FIGURE 3. Images from a series of micrographs taken by HELIOSENSOR. The white spots represent $\boldsymbol{R}$. contractilis cells. Numbers indicate time in minutes. $10 \%$ seawater was introduced into the chamber from -20 min to 0 min. Mercury solution was then introduced to the cells for $20 \mathrm{~min}$, followed by washing with $10 \%$ seawater. The number of cells attached to the substratum began to decrease at $20 \mathrm{~min}$, and most cells flowed out in $70 \mathrm{~min}$. 
For measuring axopodial length, a Nikon microscope (Eclipse Ni-U) equipped with Nomarski differential interference optics was used. Twelve individuals were measured for each experiment, and the average value of ten individuals excluding the minimum and the maximum values was taken.

For comparison, Effect of toxic substances was examined with the crustacean Artemia salina (brine shrimp) and the fish Moryzias latipes (medaka). Dried cysts of Artemia were hatched in $50 \% \mathrm{ASW}$ at $20^{\circ} \mathrm{C}$, and the 2-day-old larvae were used. Medaka fish were purchased from a local dealership, and bred for about a week before used for experiments. Artemia and medaka fish were treated with various concentrations of harmful substances, and the proportion of individuals that showed abnormal swimming behaviors after 20 min was determined as the abnormality in motility.

The experiment using the prototype monitoring device was carried out as follows. First, 10\% ASW was flowed for 10 min or more to the equipment where heliozoans were set in advance. Next, 10\% ASW containing various concentrations of toxic substances was introduced. Still images were taken continuously at intervals of $10 \mathrm{~s}$, and the number of individuals was counted by the computer. Usually 300-1,000 cells were present in the area used for measurements. Based on the number of cells before poisoning, the percentage of cells present in each image was calculated and recorded.

\section{RESULTS AND DISCUSSION}

The axopodia of $R$. contractilis is known to be shortened by various harmful substances. Fig. 1 shows the shortening of axopodia caused by treatment with $1 \mathrm{mM} \mathrm{HgCl}$ for $5 \mathrm{~min}$. Heliozoans are adhered to the substratum at the tip of axopodia. Therefore, when axopodia become shortened, the heliozoan can no longer be able to adhere to the surface and detaches from there. Thus, by measuring the length of axopodia or the adhesiveness of the cells to the substratum by axopodia, the harmfulness of the water environment can be determined quantitatively. Since axopodia of $R$. contractilis is known to react against many kinds of heavy metals [7], the device developed in the present research can detect not only mercury but also other heavy metals.

The reactivity of $R$. contractilis to heavy metals was compared with Artemia and medaka fish. As shown in Fig. 4, as a result of examining the reactivity of these organisms to mercury, arsenic, lead, and cadmium, it was found that $R$. contractilis shows the most sensitive reaction as compared with Artemia and medaka fish for all the substance. Regarding the reactivity of $R$. contractilis to mercury, the length of axopodia and the adhesion to substrate are depicted in the same graph, showing similar sensitivity in either cases. As a result, it was confirmed that $R$. contractilis can be used an organism suitable for water quality monitoring.
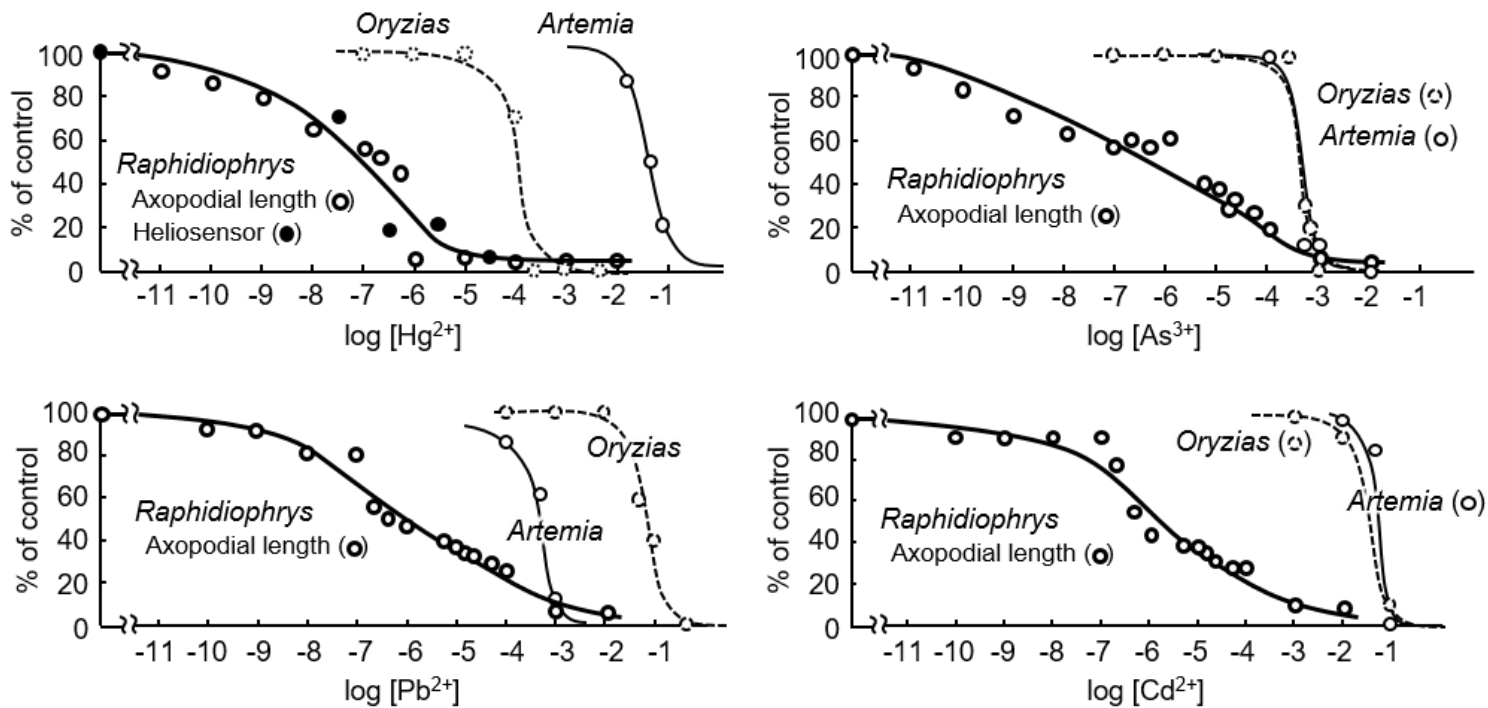

FIGURE 4. A comparison experiment performed among different monitor organisms (R. contractilis, Artemia salina (brine shrimp) and Moryzias latipes (medaka fish) on their sensitivities to heavy metals (mercury, arsenic, lead and cadmium). After 20 min exposure of heavy metals to the organisms, \% abnormality in swimming motility was examined. In $R$. contractilis, Axopodial length (open circles) and the adhesiveness to the substratum (closed circles, determined by HELIOSENSOR) became reduced by toxic substances. Brine shrimp and medaka fish showed abnormal swimming behavior and changes in their body colors when they were affected by the toxic substances. 
Fig. 5 shows an experimental result using HELIOSENSOR, by changing the concentration of mercuric chloride between $1 \times 10^{-8}$ and $1 \times 10^{-5} \mathrm{M}$. The curve of the control hardly changed. As the concentration of mercury increased, the adhesion of the cells declined. By treatment with mercury at a high concentration $\left(1 \times 10^{-5} \mathrm{M}\right)$, most cells became detached from the substratum in about $10 \mathrm{~min}$.

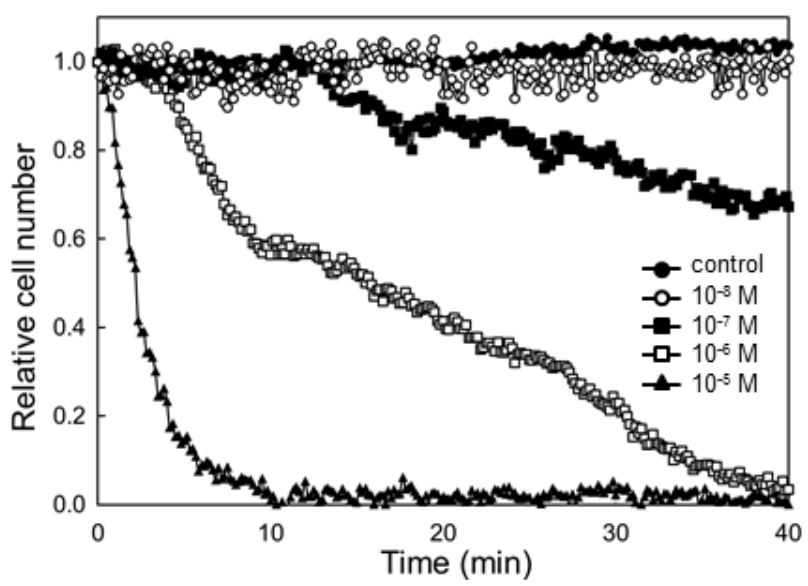

FIGURE 5. Effects of various concentration of $\mathrm{HgCl}_{2}$. The mercury solution was introduced into the chamber at time zero. Relative cell number gradually decreased, and reached almost zero at $2 \times 10^{-8} \mathrm{M}$, while at lower concentrations, the rate of decrease in cell number was dependent on the concentration of mercury ions. The vertical axis shows relative cell number, and the horizontal axis shows time (min). Images were taken at 10 second intervals and the number of cells was counted.

\section{CONCLUSION}

We have developed a water quality monitoring system for drinking water supply using protists. Since this device is highly sensitive, compact and inexpensive, it can be used in various places and situations. Moreover, this system will also be applicable to safety management such as sewage and factory wastewater.

\section{ACKNOWLEDGEMENTS}

This work was supported by JSPS KAKENHI Grant Numbers 19510030, 23117009, and 23510062.

\section{REFERENCES}

[1] Shigenaka, Y. 1976. Microtubules in protozoan cells. II. Heavy metal ion effects on degradation and stabilization of the heliozoan microtubules. Annot. Zool. Japon. 49, 164-176.

[2] Sakaguchi, M., Suzaki, T. and Shigenaka, Y. 1997. Statistical analysis of spatial patterns of the heliozoan Actinophrys sol. Protoplasma 196, 117-122.

[3] Kinoshita, E., Suzaki, T. and Shigenaka, Y. 2001. The ultrastructure of contractile tubules in the heliozoan Actinophrys sol and their possible involvement in rapid axopodial contraction. J. Euk. Microbiol. 48: 519-526.

[4] Khan, S. M. M. K., Arikawa, M. and Suzaki, T. 2005. Toxic effect of heavy metal ions on the axopodia of heliozoon Raphidiophrys contractilis. Jpn J. Protozool. 38, 44-45.

[5] Khan, S. M. M. K., Arikawa, M., Omura, G., Suetomo, Y., Kakuta, S. and Suzaki, T. 2003. Axopodial contraction in the heliozoon Raphidiophrys contractilis requires extracellular $\mathrm{Ca}^{2+}$. Zool. Sci. 20, 1367-1372.

[6] Sakaguchi, M., Suzaki, T., Khan, S. M. M. K. and Hausmann, K. 2002. Food capture by kinetocysts in the heliozoon Raphidiophrys contractilis. Europ. J. Protistol., 37, 453-458.

[7] Khan, S. M. M. K., Yoshimura, C., Arikawa, M., Omura, G., Nishiyama, S., Suetomo, Y., Kakuta, S. and Suzaki, T. 2006. Axopodial degradation in the heliozoon Raphidiophrys contractilis: A novel bioassay system for detecting heavy metal toxicity in the aquatic environment. Environ. Sci. 13, 193-200.

[8] Jeong, Y. T., Jeon, J. and Kim, D. S. 2014. Development and evaluation of new behavioral indexes for a biological early warning system using Daphnia magna. Drink. Water Eng. Sci. 7, 1-9.

[9] Kang, J. I., Moroishi, J., Nakamura, A., Nagafuchi, K., Kim, G. S. and Oshima, Y. 2009. Biological monitoring of detection of toxic chemicals in water by the swimming behavior of small freshwater fish. Journal of the Faculty of Agriculture Kyushu University, 5 , 209-214.

[10] Liao Y., Xu, J., and Wang, Z. 2012. Application of biomonitoring and support vector machine in water quality assessment. J Zhejiang Univ-Sci B (Biomed \& Biotechnol), 13, 327-334. 